\title{
Calidad percibida en una prueba de triatlón
}

\author{
Perceived quality in a triathlon
}

\author{
Angosto Sánchez, S. ${ }^{1 *}$, Morán-Navarro, R. ${ }^{1}$, Martínez-Cava, A. ${ }^{1}$ y López-Gullón, J.M. \\ ${ }^{1}$ Facultad Ciencias del Deporte. Universidad de Murcia.
}

\begin{abstract}
Resumen: El interés por conocer las percepciones del participante respecto a la calidad y el valor del servicio, se ha trasladado a los eventos deportivos de carácter popular. Conocer los elementos determinantes para alcanzar la satisfacción general del participante, propiciará a los organizadores una mayor fidelización y mejora enpróximos eventos. A través de un cuestionario, este estudio analiza las distintas dimensiones de la calidad que presenta una prueba de triatlón sobre valor percibido y las intenciones futuras según la percepción del corredor participante en el "XXVI Triatlón nacional Villa de Fuente Álamo". Los resultados mostraron la dimensióninfraestructura logística como la mejor valorada dentro de los factores de calidad y la dimensión comunicación como la peor valorada. Los participantes con una mayor experiencia obtuvieron puntuaciones más bajas.
\end{abstract}

Palabras Clave: Triatlón, eventos deportivos, calidad del servicio, valor percibido.

Abstract: The interest in knowing participant perceptions regarding the quality and value of the service, has moved to sports events popular character. Knowing what are the determining factors for achieving the overall participant satisfaction, the organizers will favor greater loyalty and improved planning of upcoming events. Through a questionnaire, this study analyzes the different dimensions of quality, which has a triathlon, the perceived value and future intentions as perceived by the participant broker in the "XXVI National Triathlon Villa de Fuente Álamo». The results show that the logistics infrastructure dimension as the most valued factor in quality and communication the worse punctuation. The participants with greater experience obtained lower scores than the other groups.

Keywords: Triathlon, sporting event, service quality, perceived value.

\section{Introducción}

El incremento de la práctica deportiva de la población residente en España en las tres últimas décadas ha sido del 15\%, la tasa ha sufrido un incremento desde el $25 \%$ en 1980 al $40 \%$ en 2010, siendo mayor, del 18\%, el incremento con respecto al ańo 1975 (García y Llopis, 2010). Esta gran demanda social, ha provocado la aparición de un amplio número de empresas que buscan cubrir esas necesidades, no sin sufrir un crecimiento adaptativo para satisfacer las demandas y cubrir las expectativas de los deportistas ante una oferta tan amplia.

Este aumento de práctica deportiva, considerando la situación económica mundial actual, provoca que la actividad física se realiza principalmente de forma popular, acercándose cada vez más a los diferentes niveles sociales. Esto se traduce en mayores expectativas y posibilidades de participación por parte del usuario ante lo que las empresas deben saber responder y satisfacer (Bodet, 2006; Dorado y Gallardo, 2004; Kim y Kim, 1995; Luna-Arocas y Li-Ping, 2005; Murray y Howat, 2002; Robinson, 2006).

Ante tanta variedad, se fomenta un espíritu crítico entre los participantes, esto hace que la calidad se esté convirtiendo en una necesidad esencial a la hora de elaborar estrategias que les permitan diferenciarse como empresa dentro de un mercado altamente competitivo (Gálvez y Morales-Sánchez, 2011).

Disponer de un eventoque ofrezca una mejor experiencia deportiva que la competencia hace que hoy en día el concepto de calidad tenga una importancia vital para todo el proceso

Dirección para correspondencia [Correspodence address]: S. Angosto Sánchez. E-mail: salvador.a.s@um.es de desarrollo y comercialización dentro de las empresas deportivas. De esta forma, la enorme, creciente y heterogénea afluencia de público que se genera en torno a los acontecimientos deportivos de la actualidad hace que resulte esencial profundizar en el comportamiento de los usuarios e identificar sus necesidades y demandas de cara al desarrollo de adecuados planes de calidad que aporten eficacia y eficiencia en la gestión de las organizaciones (Gálvez, Morales, Hernández, Pérez y López, 2012).

Por lo tanto la calidad se ha convertido en una necesidad en las empresas para poder garantizar su continuidad y progreso, considerando la calidad como un punto de diferenciación dentro del sector generando mayor beneficio y satisfacción a los participantes, organizadores, dirigentes y la propia imagen de la localidad. Centrarse en mejorar la calidad y la satisfacción del cliente es lo más importante para que se desarrollen y consoliden las organizaciones (Afthinos, Theodorakis y Nassis, 2005; Westerbeek y Shilbury, 2003), y a la misma vez, se incrementa y mejora la fidelización de los clientes (Calabuig, Burillo, Crespo, Mundina y Gallardo, 2010).

La calidad percibida, se puede considerar como el "juicio global, o actitud, relativa a la superioridad del servicio” (Parasuraman, Zeithaml y Berry, 1988).

Analizar el perfil del consumidor, así como sus necesidades y demandas, es un importante campo de estudio y un factor determinante en actualidad para llegar a ser competitivos (Mármol, Orquín Castrillón y Sáinz de Baranda, 2010). En palabras de Barroso, Martín y Martin (2004), la capacidad de crear y entregar valor al cliente ha sido denominada 
como la fuente más importante de ventaja competitiva del siglo XXI.

El valor percibido ha pasado a ser clave en investigaciones de marketing. Justamente, es un concepto que dispone de la aprobación de investigadores debido a los beneficios que se derivan de la generación y entrega de valor a los clientes (Barroso et al., 2004).

El deporte y los eventos asociados a éste o cualquier forma de actividad física son un factor de amplia repercusión social en la sociedad actual. Los eventos deportivos están caracterizados por un nivel de incertidumbre, es decir, el nivel de duda que ronda en torno a factores clave para su realización como la cantidad de participantes, el coste total o la información que sobre el propio evento se debe facilitar y como hacerla llegar al público (Cerezuela, 2003).Para que la organización de una eventualidad deportiva se lleve a cabo de manera eficaz se debe actuar en base a una serie de pautas o estándares que nos permitan disminuir los posibles factores de riesgos asociados a dicho evento.

La propia naturaleza del triatlón hace que el perfil difiera respecto a otras disciplinas, y es por ello que la variedad deportiva y el reto/aventura planteada por el triatlón (ligado a la práctica en entornos naturales), inexistente en otros deportes, suponga la principal motivación para el inicio de la práctica (Ruiz-Tendero, Salinero y Sánchez Bañuelos, 2008).

La experiencia deportiva debe ser un elemento de ocio más para el consumidor, que valora la correcta prestación de servicios para su disfrute. Así, el indicador futuro que toda empresa debe buscar, es la fidelización de sus clientes y ofrecer un valor ańadido a sus productos o servicios (Tsitskari, Tsiotras y Tsiotras, 2006).

Según Calabuig, Mundina y Crespo (2010) la satisfacción se postula como una antecedente del comportamiento futuro del cliente. La importancia de la satisfacción es que, puede considerarse como el paso predecesor a la fidelidad de un cliente (Murray y Howat, 2002). Además, un cliente satisfecho es propenso a compartir su experiencia.

Calabuig et al. (2010) y Theodorakis Kambitsis, Laios y Koustelios (2001), en referencia a la satisfacción de los espectadores, concluyen que los elementos tangibles y el valor monetario percibido por los espectadores de un gran evento deportivo son determinantes para explicar su satisfacción general.

En definitiva, analizar el nivel de satisfacción de los espectadores y participantes, así como el de las distintas dimensiones de calidad y valor del servicio percibido facilitará a los gestores la detección de posibles carencias y la adecuación de manera correcta sus servicios a la demanda existente (Calabuig, et al. 2010).

El objetivo principal de este estudio es conocer y evaluar la calidad percibida de los participantes que tomaron parte de la XXVI edición del Triatlón nacional Villa de Fuente de Álamo en función de la experiencia previa de la misma.
Como objetivo que nace del anterior se encuentra con el afán por conocer la opinión de los triatletas sobre la prueba en general y sobre sus peculiaridades en cuanto a organización y ejecución; para finalmente obtener una idea general desde el punto de vista del participante que ayude a la comisión organizadora de esta prueba a modificar aquellas cosas que no funcionaron del todo bien y mejorar aquellos aspectos que si salieron correctamente.

\section{Método}

\section{Muestra}

La muestra estudiada está formada por 147 participantes en la XXVI edición del Triatlón Nacional Villa de Fuente Álamo de un total de 800 participantes inscritos (18'4\%). El sexo de los participante fue predominantemente de hombres, 120 varones $\left(81^{\prime} 6 \%\right)$ y 27 mujeres (18'4\%) mujeres. La edad media de los participantes es de 34 '7 años (+8'86). El 25,9\% de los participantes cuentan con una experiencia previa de más de 10 años frente al 8,2\% que participa en este tipo de pruebas desde hace menos de un año o que se inician en esta. El 37,4\% de los participantes dedican entre 6-10 horas de práctica de este deporte a la semana, seguido de un 31,3\% que le dedica más de 10 horas y solo un 10,2\% le dedica menos de 3 horas La mayoría de los corredores tienen estudios universitarios superiores (43'5\%) o medios (21'8\%) no encontrando ningún corredor que no tuviera estudios. La mayoría de participantes se encuentran en situación laboral activa $\left(84^{\prime} 4 \%\right)$ mientras que solo el 6' $8 \%$ son desempleados.

\section{Instrumento}

El cuestionario utilizado, sirve para valorar la calidad percibida y es una adaptación del cuestionario diseñado por Angosto, López-Gullón, Martínez-Moreno (2014) para carreras populares, compuesto de 22 ítems, al que se le han ańadido 10 ítems para la modalidad de triatlón. Este instrumento está compuesto, por un total de 6 dimensiones de calidad, atendiendo a los aspectos generales que debe disponer un evento popular, como un triatlón. La alternativa de respuesta es de tipo Likert de seis anclajes (1 - Muy en desacuerdo a 6-Muy de acuerdo).

\section{Propiedades psicométricas}

Para comprobar la validez del cuestionario propuesto, se aplicaron los tratamientos de fiabilidad y validez. La fiabilidad (cálculo de la consistencia interna de la escala), se determina a través del coeficiente alfa de Cronbach, obteniendo todos los factores índices adecuados en la mayoría de dimensiones (Tabla 1). 
Tabla 1. Fiabilidad de los factores y escala.

\begin{tabular}{lcc}
\hline Escala & Alfa de Cronbach & No de Ítems \\
\hline Comunicación & .519 & 3 \\
Infraestructura logística & .800 & 5 \\
Interacción personal & .807 & 4 \\
Servicios complementarios & .734 & 4 \\
Box-Transición & .802 & 6 \\
Aspectos técnicos & .802 & 6 \\
\hline Total dimensiones & .930 & 28 \\
\hline
\end{tabular}

\section{Procedimiento de recogida de datos}

La administración del cuestionario a los participantes, se realizó de forma telemática, mediante la aplicación del cuestionario de forma online. Se puso en contacto con los organizadores de la prueba solicitando la base de datos de los participantes donde figuraban todos los correos electrónicos y se pidió permiso para pasar el cuestionario con el fin de medir la calidad de la prueba.

\section{Análisis de datos}

El análisis de los datos se realizó, con el paquete estadístico SPPS 19 con licencia de la Universidad de Murcia. Las pruebas que se hicieron, fue análisis descriptivo y una ANOVA para observa la posible existencia de diferencias significativa entre grupos y el Alfa de Cronbach para calcular la fiabilidad del instrumento.

\section{Resultados}

El análisis de resultados se estableció una comparativa de las medias de las diferentes dimensiones de la escala de calidad percibida en función de la experiencia previa de los corredores (Tabla 2). La experiencia previa fue agrupada en tres grupos: participantes con menos de tres ańos de experiencia (34,7\%), entre 3 y 10 años de experiencia $(39,5 \%)$ y más de 10 ańos de experiencia en pruebas de este tipo (25,8\%). El grupo de más de 10 años de experiencia fue el que obtuvo menores puntuaciones.

Tabla 2. Estadísticos descriptivos y ANOVA de la escala de calidad percibida.

\begin{tabular}{|c|c|c|c|c|c|c|c|c|c|}
\hline \multirow{2}{*}{$\begin{array}{l}\text { Experiencia Previa } \\
\text { DIMENSIONES }\end{array}$} & \multicolumn{2}{|c|}{ Menos de 3 años } & \multicolumn{2}{|c|}{ De 3 a 10 años } & \multicolumn{2}{|c|}{ Más de 10 años } & \multicolumn{2}{|c|}{ TOTAL } & \multirow{2}{*}{ Sig } \\
\hline & M & D.T. & M & D.T. & M & D.T. & M & D.T. & \\
\hline Comunicación & 4,91 & ,79 & 4,69 & ,87 & 4,77 & ,78 & 4,79 &, 81 &, 372 \\
\hline Infraestructura logística & 5,61 &, 57 & 5,58 &, 55 & 5,65 &, 52 & 5,61 &, 54 &, 802 \\
\hline Interacción Personal & 5,28 &, 65 & 5,03 &, 78 & 5,17 &, 74 & 5,15 &, 73 & ,200 \\
\hline Servicios complementarios & 4,80 & ,69 & 4,94 &, 87 & 5,07 & 1,00 & 4,93 &, 85 &, 322 \\
\hline Box-Transición & 5,39 &, 53 & 5,30 &, 61 & 5,37 &, 550 & 5,35 &, 57 & ,697 \\
\hline Aspectos Técnicos & 5,36 &, 54 & 5,31 &, 54 & 5,35 &, 474 & 5,33 &, 52 &, 865 \\
\hline
\end{tabular}

Los resultados arrojaron que para todos los grupos el factor mejor valorado fue la infraestructura logística, al igual que en la valoración global de todos los grupos. Resultados coincidentes con los de Calabuiget al. (2010) sobre espectadores en pruebas de atletismo cuya dimensión mejor valorada fueron los elementos tangibles.

Los factores propios del triatlón de box-transición y de aspectos técnicos también se encuentran con altas valoraciones, todas por encima de 5 puntos sobre la escala Likert y resultados similares para los tres grupos, además en la valoración global se tratan de la segunda y tercera dimensión mejor de la escala valorada con un 5,35 y 5,33 respectivamente.

Por el contrario, existe una mayor discrepancia con los factores peor valorados por los participantes. Para aquellos con menos de tres años de experiencia previa fueron los servicios complementarios $(4,80)$, en participantes que contaban entre 3 y 10 años de experiencia $(4,69)$ y en los de más de 10 años $(4,77)$ fue la dimensión comunicación., igual que la valoración total.
Se aplicó una prueba ANOVA resultando que no existieron diferencias significativas en ninguna de las dimensiones.

\section{Conclusiones}

Las principales conclusiones que se pueden extraer de este estudio son:

- Los participantes con una experiencia de más de 10 años fueron los más críticos al obtener unas valoraciones más bajas en todas las dimensiones.

- La dimensión mejor valorada por cada grupo y en general fue la de infraestructura logística, siendo la peor valorada la comunicación.

- Las dimensiones específicas del triatlón (box-transición y aspectos técnicos) obtuvieron puntuaciones altas y muy similares en todos los grupos. 


\section{Referencias}

1. Afthinos, Y., Theodorakis, N. D., y Nassis, P. (2005). Customers' expectations of service in greek fitness centers. gender, age, type of sport center, and motivation differences. ManagingServiceQuality, 15(3), 245 258 .

2. Angosto, S., López-Gullón,J.M., y Martínez-Moreno, A. (2014). Diseño de contenido de un cuestionario sobre la calidad percibida por el participante en carreras populares. En Espitia, M.A., García-Cebrián, L.I., Muñoz-Pocar, A., Serrano-Lázaro, R. y Gutiérrez-Ilarduya, J. (Eds.), V Congreso Iberoamericano de Economía del Deporte. El deporte: Dinamizador económico (p. 19-22). Zaragoza, España

3. Barroso Castro, C., Martín Armario, E., \& Martín Ruiz, D. (2004). The influence of employee organizational citizenship behavior on customer loyalty.International journal of Service industry management, 15(1), 27-53.

4. Bodet, G. (2006). Investigating customer satisfaction in a health club context by an application of the tetraclasse model.European Sport Management Quarterly, 6(2), 149-165.

5. Calabuig, F., Mundina, J. J., \& Crespo, J. (2010).Eventqual: Una medida de la calidad percibida por los espectadores de eventos deportivos. [Versión electrónica]. Retos. Nuevas Tendencias En Educación Física, Deporte y Recreación, 18, 66-70.

6. Calabuig, F.; Burillo, P.; Crespo, J.; Mundina, J.J. \& Gallardo, L. (2010). Satisfacción, calidad y valor percibido en espectadores de atletismo. [Versión electrónica]. Revista Internacional de Medicina y Ciencias de la Actividad Física y el Deporte, 10 (40), 577-593.

7. Cerezuela, B. (2003). La información y documentación deportiva y los grandes eventos deportivos. Barcelona: Centro de Estudios Olímpicos, Universidad Autónoma de Barcelona.

8. Dorado A. y Gallardo L. (2004). Gestión del deporte a través de la calidad. Barcelona: INDE.

9. Gálvez, P. \& Morales Sánchez, V. (2011). Evaluación de la calidad en programas municipales deportivos: generalizabilidad y optimización de diseños de medida. [Versión electrónica]. Cuadernos de Psicología del Deporte, 11(2), 123-130.

10. Gálvez, P., Morales-Sánchez, V., Hernández-Mendo, A., Pérez-López, R. y López-Linares, A. (2012). Evaluación en gestión deportiva: calidad de servicios. Revista de Ciencias del Ejercicio FOD, 7(7), 29-37.

11. García Ferrando, M., \&LlopisGoig, R. (2011). Encuesta sobre los hábitos deportivos en España 2010. Ideal democrático y bienestar personal.

12. Kim, D., y Kim, S. Y. (1995). QUESC: an instrument for assessing the service quality of sport centers in Korea. Journal of Sport Management, 9(2), 208-220.

13. Luna-Arocas, R., y Li-Ping, T. (2005). The use of cluster analysis to segment clients of a sport center in Spain. European Sport Management Quarterly, 5(4), 381-413.

14. Marmol, A., Orquín Castrillón, F. J., \& Sainz de Baranda, P. (2010). La infraestructura y el equipamiento, la prescripción del ejercicio y los servicios ofertados como índices de calidad de los centros fitness de Murcia. Cuadernos de Psicología del Deporte, 10(2), 85-91.

15. Murray, D. \& Howat, G (2002). The relationships among service quality, value, satisfaction, and future intentions of customers at an Australian sports and leisure centre. Sport Management Review. 2002; 5:25-43.

16. Parasuraman, A., Zeithaml, V. y Berry, L. (1985). A conceptual model of service quality and its implications for future research.Journal of Marketing, 49, 41-50.

17. Robinson, J. (2006). Ordinary cities: between modernity and develop ment (Vol. 4). Psychology Press.

18. Ruíz-Tendero, G., Salinero, J., y Sánchez-Bañuelos, F. (2008). Valoración del perfil sociodemográfico en el triatleta: el ejemplo de Castilla-la Mancha: Nivel de implicación y entorno. Apunts. Educación Física y Deportes, 92, 5-14.

19. Theodorakis, N., Kambitsis, C., Laios, A. \&Koustelios, A. (2001). Relationship between measures of service quality and satisfaction of spectators in professional sports.Managing Service Quality, 11(6), 431-438.

20. Tsitskari, E., Tsiotras, D., \& Tsiotras, G. (2006).Measuring service quality in sport services.Total Quality Management and Business Excellence, 17(5), 623-631.

21. Westerbeek, H. M., y Shilbury, D. (2003). A Conceptual Model for Sport Services Marketing Research: Integrating Quality, Value and Satisfaction. International journal of sports marketing \& sponsorship, 5(1), $11-31$. 